\title{
ÚLTIMAS TENDENCIAS EN LA PRODUCCIÓN CIENTÍFICA EUROPEA EN CIENCIAS SOCIALES, COMUNICACIÓN Y HUMOR FIGURA(2007-2011)
}

Francisco Segado-Boj 1: Universidad Internacional de la Rioja. España francisco.segado@unir.net

\section{RESUMEN}

Esta investigación analiza las tendencias existentes en la producción científica europea en Ciencias Sociales que toma como objeto de estudio el humor gráfico. Para ello se han analizado los datos disponibles en el ISI Web of Knowledge sobre los artículos científicos publicados al respecto en esta base de datos de referencia. Con estos datos se ha analizado esta producción científica por autores, instituciones y países de procedencia, así como por las áreas de interés a la que se adscriben las revistas en las que se han incluido esos artículos. Se ha prestado también especial atención al impacto de cada uno de estos trabajos, medido en el número de citas recibido por cada artículo. De este modo se ha demostrado que la Comunicación muestra un especial interés hacia este objeto de estudio que no se traduce en un número de citas parejo.

PALABRAS CLAVE: Comunicación - Europa - Humor Figura- Investigación

\section{LATEST TRENDS IN THE EUROPEAN SCIENTIFIC PRODUCTION IN SOCIAL SCIENCIES, COMMUNICATION AND POLITICAL CARTOONS (2007-2011)}

\begin{abstract}
This paper analyses current trends in the Social Sciences European production focused on political cartoons. In order to do so, data available from ISI Web of Knowledge regarding papers published about this topic has been analyzed. Information about this scientific production has been categorized by authors, as well as by their affiliation and country. Journals subject areas have also been considered.

\footnotetext{
${ }^{1}$ Autor correspondiente

Francisco Segado-Boj: Profesor Adjunto. Universidad Internacional de la Rioja. Logroño, España

Correo: francisco.segado@unir.net
} 
Special attention has been paid as well to the impact of these papers, measured by the number of quotes achieved by each of them. So it has been unveiled that Communication shows a special interest towards this object of study, which is not accompanied by a high quotation index.

KEY WORDS: Communication - Europe - Political cartoons - Research

\section{INTRODUCCIÓN}

El estudio del humor Figuraha inspirado desde hace años numerosos trabajos en distintas disciplinas como es el caso de la Historia del Arte (Bozal, 1989), la Lingüística (Llera, 2003) o la Historia Contemporánea (Zeman, 1987). Las Ciencias Sociales también han dirigido su atención a este objeto de estudio, desde disciplinas como la Educación (Guitart-Coria y Flores Martínez, 2003). Por supuesto la Comunicación no ha sido ajena a esta tendencia y ha ofrecido estudios desde campos como la Semiótica (Peñamarín, 2002) o la Historia de la Prensa (Tubau, 1987).

El objetivo de este estudio consiste en identificar la situación en la que se encuentra la producción científica sobre humor Gráfico en la actualidad en los países que constituyen el Espacio Europeo de Educación Superior. Para ello se han analizado cuantitativamente los artículos científicos publicados sobre este asunto en los últimos cinco años. Se ha tomado como referencia esta modalidad de difusión de la actividad investigadora en lugar de otras opciones como las monografías porque las revistas científicas "en tanto que publicaciones periódicas constituyen el vehículo adecuado para la presentación de la investigación última, pues el lapso entre producción y publicación no suele demorarse en exceso. Son, por tanto, el indicador más fiable de las líneas de investigación abiertas, y allí se expresan de la manera más actualizada los intereses de conocimiento de la comunidad científica en un momento determinado" (Martínez Nicolás y Saperas Lapiedra, 2011, p. 105).

El último objetivo de este trabajo consiste en señalar cuáles son los temas que despiertan una mayor atención dentro de todos aquellos tratados por los artículos analizados. Para ello se ha cuantificado el número de citas recibidas por estas publicaciones según los datos recogidos por el mismo ISI.

\section{METODOLOGÍA}

Para ceñir el estudio a la investigación académica de calidad se ha delimitado el cuerpo del estudio a artículos publicados en revistas indexadas en la base de datos Web of Knowledge del Institute for Scientific Information (http://www.webofknowledge.com). La búsqueda se ha restringido a artículos publicados en los últimos cinco años para detectar y poder señalar las tendencias que están configurando el panorama de estas investigaciones en el marco del Espacio Europeo de Educación Superior. El análisis se ha ceñido a artículos científicos, por lo que se han eliminado las reseñas sobre libros de la muestra. Dentro de la base de datos han sido seleccionados aquellos trabajos que en su título y sus palabras claves 
empleasen los términos "political cartoon" o "political cartoons", por lo que se ha empleado el término de búsqueda "political cartoo*".

El análisis de la muestra obtenida tras esta selección permite descubrir las directrices existentes dentro del panorama científico sobre el humor Figuray diagnosticar su situación actual. De modo más concreto este estudio pretende desentrañar cuáles son los autores, países e instituciones que han publicado un mayor número de artículos al respecto.

Así mismo también analiza los campos del conocimiento en los que se ha mostrado un interés más amplio respecto al humor gráfico. Para ello, se ha atendido al área o áreas de conocimiento al que el ISI Web of Knowledge asigna a las revistas en las que se ha publicado cada artículo. La única excepción ha sido la revista Discourse, que aparece sin ubicarse en una categoría concreta. La web oficial de esta publicación (http://www.tandf.co.uk/journals/cDIS) ${ }^{2}$ especifica que está dedicada "a las políticas culturales sobre educación". Por ello se ha incluido esta revista en el campo de “Educación".

\section{ANÁLISIS Y DISCUSIÓN}

\subsection{Panorama global de la investigación sobre humor Gráfico en Ciencias Sociales}

De los 34 artículos sobre humor Figurapublicados entre 2007 y 2011 que se encuentran listados en el ISI Web of Knowledge, catorce de ellos han sido publicados por autores vinculados a instituciones de países integrados en el Espacio Europeo de Educación Superior. Este dato representa el $41 \%$ de la producción científica mundial sobre este tema (ver Figura1).

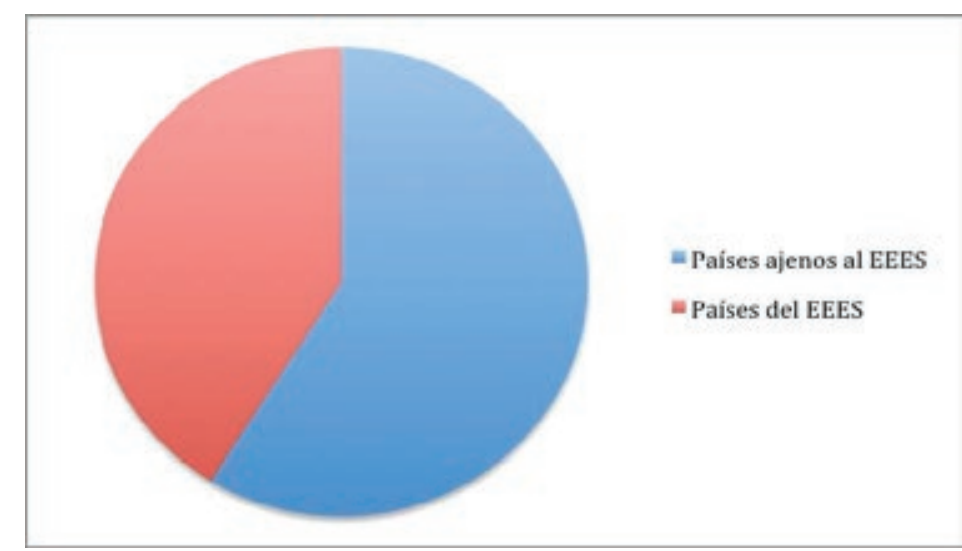

Figura1. Porcentaje de artículos sobre humor gráfico publicado por países incluidos en el E.E.E.S. y ajenos al E.E.E.S.

\footnotetext{
$\overline{2}$ Consultada el 18 de Enero de 2011.
} 
Los catorce títulos mencionados en el ISI Web of Knowledge se listan en la Tabla 1, que aparece a continuación ${ }^{3}$

Tabla 1. Artículos sobre humor gráfico publicados por autores del E.E.E.S. (20072011)

\begin{tabular}{|c|c|c|}
\hline Título del artículo & Autor/es & Año \\
\hline "Resistance, power and geopolitics in Zimbabwe" & Hammett, Daniel & 2011 \\
\hline $\begin{array}{l}\text { "Metaphors in editorial cartoons representing the global } \\
\text { financial crisis" }\end{array}$ & $\begin{array}{l}\text { Bounegru, Liliana; } \\
\text { Forceville, Charles }\end{array}$ & 2011 \\
\hline "Beyond Decoding: Political Cartoons in the Classroom" & $\begin{array}{l}\text { Hammett, Daniel; } \\
\text { Mather, Charles }\end{array}$ & 2011 \\
\hline “Don't take it personally, it's just a joke: & $\begin{array}{l}\text { Aliefendioglu, Hanife; } \\
\text { Arslan, }\end{array}$ & 2011 \\
\hline $\begin{array}{l}\text { The masculine media discourse of jokes and cartoons on } \\
\text { the Cyprus issue" }\end{array}$ & Yetin & 2011 \\
\hline $\begin{array}{l}\text { "Crisis Communication as a Multilevel Game: The } \\
\text { Muhammad Cartoons from a Crisis Diplomacy } \\
\text { Perspective" }\end{array}$ & $\begin{array}{l}\text { Lindholm, Kristina; } \\
\text { Olsson, Eva-Karin }\end{array}$ & 2011 \\
\hline $\begin{array}{l}\text { "Young people's readings of a political cartoon and the } \\
\text { concept of multimodal literacy" }\end{array}$ & $\begin{array}{l}\text { El Refaie, Elisabeth; } \\
\text { Hoerschelmann, } \\
\text { Kathrin }\end{array}$ & 2010 \\
\hline $\begin{array}{l}\text { “El brazo informativo del gobierno: TVE en el humor } \\
\text { Figurade la prensa diaria de la transición (1974-1977)" }\end{array}$ & Segado, Francisco & 2009 \\
\hline $\begin{array}{l}\text { "Multiliteracies: how readers interpret political } \\
\text { cartoons" }\end{array}$ & El Refaie, Elisabeth & 2009 \\
\hline $\begin{array}{l}\text { "Una pesadilla obsesiva: la crisis económica y la } \\
\text { transición española a la democracia a través del humor } \\
\text { Figura(1974-1977)" }\end{array}$ & Segado, Francisco & 2008 \\
\hline $\begin{array}{l}\text { "Elenco de una nueva etapa: los protagonistas de la } \\
\text { transición en las viñetas de la prensa diaria" }\end{array}$ & Segado, Francisco & 2008 \\
\hline "Cognition and culture in political cartoons" & Martín-Arrese, Juana I. & 2008 \\
\hline $\begin{array}{l}\text { "About face: Facial prominence of George W. Bush in } \\
\text { political cartoons as a function of war" }\end{array}$ & $\begin{array}{l}\text { Calogero, Rachel M.; } \\
\text { Mullen, Brian }\end{array}$ & 2008 \\
\hline $\begin{array}{l}\text { "The Erotic and the Vulgar: Visual Culture and } \\
\text { Organized Labor's Critique }\end{array}$ & Gerteis, Christopher & 2007 \\
\hline
\end{tabular}

Fuente: ISI Web of Knowledge.

Se puede observar que la presencia del humor gráfico en los artículos incluidos en el ISI Web of Knowledge goza de cierta constancia en los últimos años, ya que en ninguno de los cinco años abarcados por este estudio el número de artículos publicados al

${ }^{3}$ La referencia completa de estos artículos puede encontrarse en el apartado 8: Bibliografía. 
respecto ha descendido a cero. Pese a que en 2009 y 2010 el número de artículos sobre este tema fue relativamente bajo, en 2011 se ha experimentado un aumento en la producción científica sobre la materia. Este dato podría indicar una tendencia al alza en el interés que este objeto de estudio despierta entre los investigadores europeos.

Tabla 2. Número de artículos publicados sobre humor gráfico por año

\begin{tabular}{|l|l|}
\hline Año & $\begin{array}{l}\text { Número de } \\
\text { artículos }\end{array}$ \\
\hline 2007 & 2 \\
\hline 2008 & 4 \\
\hline 2009 & 2 \\
\hline 2010 & 1 \\
\hline 2011 & 5 \\
\hline
\end{tabular}

\subsection{Tendencias y rasgos de la investigación europea sobre humor gráfico}

\subsubsection{Nacionalidad: el aplastante dominio de Reino Unido}

El Reino Unido acapara la mayor parte de artículos publicados sobre humor gráfico. El 50\% de estas obras aparece firmada por autores vinculados a instituciones de este país.

Esta predominancia de autores de universidades británicas se explica en parte por la "preponderancia en el sistema investigador de las publicaciones científicas anglosajonas (...) que limita sobremanera el grado de influencia de las revistas científicas no pertenecientes a estas empresas editoriales" (Castillo Esparcia y Ruiz Mora: 2011, p. 8).

Pese a este sesgo a favor del área anglosajona se puede observar una significativa presencia de autores ligados a instituciones españolas, que firman un total de cuatro artículos. El resto de países (Holanda, Suecia y Turquía) sólo aportan un artículo a la muestra, como puede observarse en el Figura 2. 


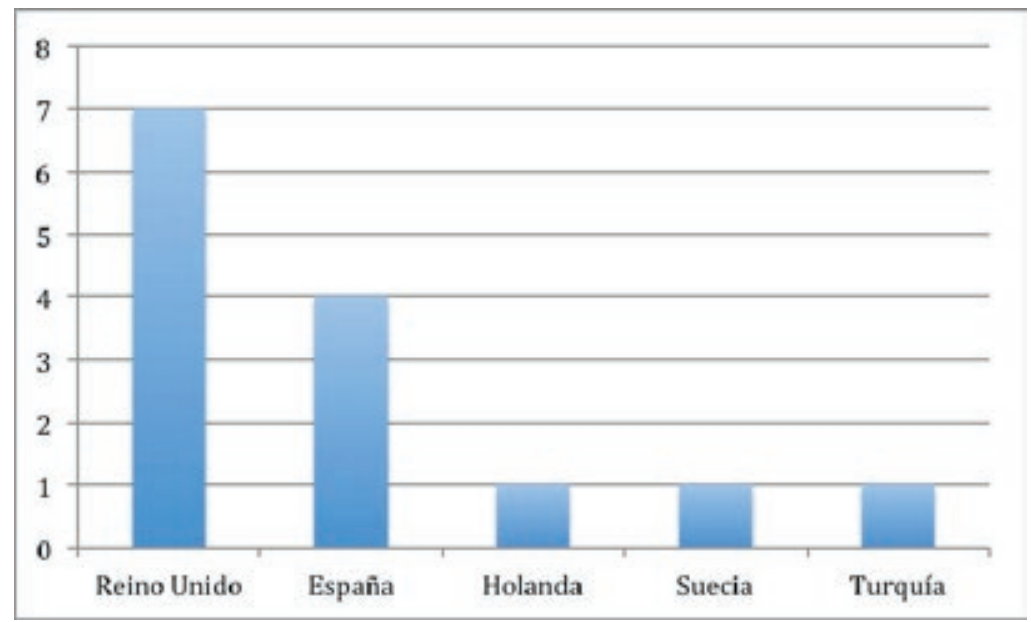

Figura 2. Número de artículos sobre humor gráfico publicados entre 2007 y 2011 por país de procedencia de la institución del autor

\subsubsection{Filiación institucional: la ligera ventaja de la Universidad Complutense}

Al desglosar la vinculación de los autores a instituciones concretas y no por países se puede observar una presencia destacada de la Universidad Complutense, cuyos investigadores firman un mayor número de artículos.

Salvo tres excepciones (Universidad de Londres, Universidad de Cardiff y Universidad de Sheffield), el resto de instituciones sólo aparecen en una única ocasión (ver Figura 3)

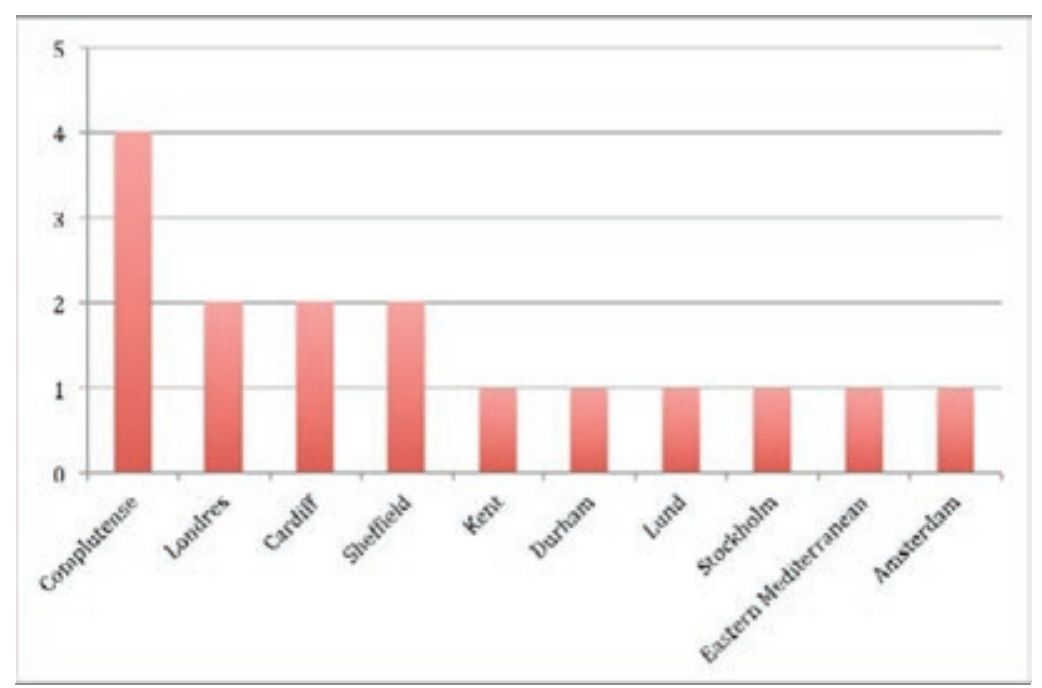

Figura 3. Número de artículos sobre humor gráfico publicados entre 2007 y 2011 por filiación insitucional del autor firmante.

\subsubsection{Autoría individual: un archipiélago de autores y obras aisladas}

En cuanto a los autores, sólo tres de ellos firman más de un artículo de los que aparecen en el ISI Web of Knowledge. El autor más prolífico es Francisco Segado quien 
con sus tres textos se sitúa como el autor más productivo sobre humor gráfico en los últimos cinco años.

Los dos siguientes autores con más presencia en la muestra son Daniel Hammett y Elisabeth El Refaie, que firman dos artículos cada uno de ellos (ver Figura 4).

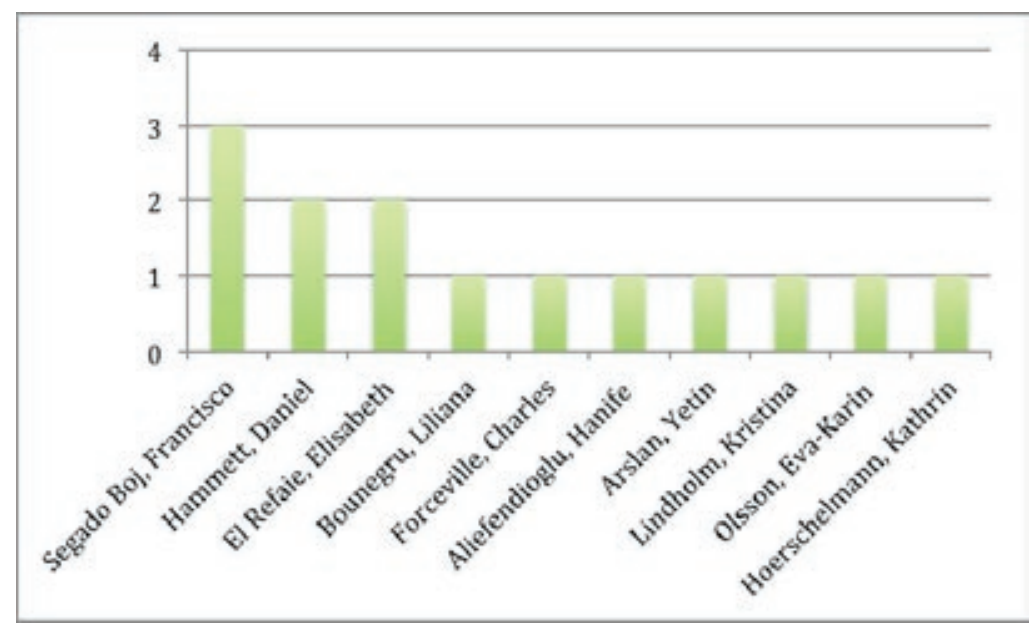

Figura 4. Número de artículos por autor (2007-2011)

Fuente: ISI Web of Knowledge.

\subsection{Impacto de la investigación europea sobre humor gráfico}

De los catorce artículos que componen la muestra, tan sólo seis han recibido citas en otros trabajos, según los datos ofrecidos por el ISI Web of Knowledge (ver Figura 5). El artículo más citado es "Steve Bell's eye: Cartoons, geopolitics and the visualization of the "War on terror'" de Klaus Dodds, un estudio que analiza la visión ofrecida por el editorialista gráfico del rotativo británico The Guardian, Steve Bell sobre la "Guerra contra el terror" de la administración de George Bush (Dodds, 2007. Pág 157).

Este artículo es mencionado en otros dieciséis trabajos. Esta cifra le sitúa a una considerable distancia del resto de estudios de la muestra, que apenas reciben una o dos citas en el mejor de los casos

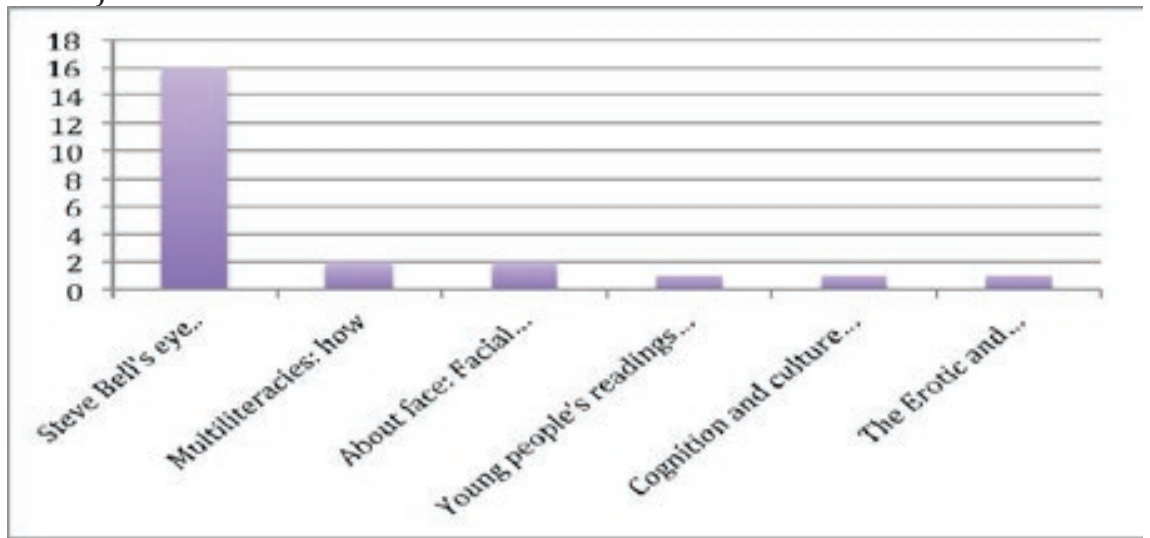

Figura 5. Número de citas recibidas por los artículos sobre humor gráfico publicados entre 2007 y 2011 
De estos artículos, dos de ellos se centran el proceso de recepción del humor gráfico. El primero de ellos ("Multiliteracies: how readers interpret political cartoons", de Elisabeth El Refaie) consigue dos citas y aborda la descodificación del mensaje contenido en los chistes y viñetas (El Refaie, 2009. Pág. 181). La misma autora ha publicado junto con Kathrin Hörschelmann otro artículo sobre el mismo tema que ha sido citado en una ocasión. Este trabajo se centra en el proceso de recepción de chistes gráficos y viñetas en un grupo de jóvenes británicos entre 16 y 19 años (El Refaie y Hörschelmann, 2010. Pág. 195).

Otro de los artículos citados es el de Marín-Arrese "Cognition and culture in political cartoons". Este estudio concreto se centra en los mecanismos de construcción de significado (Marín Arrese, 2008. Pág. 1). Este artículo cuenta con una cita, al igual que el de Rachel M. Calogero y Brian Mullen, "About face: Facial prominence of George W. Bush in political cartoons as a function of war". Este otro artículo analiza las caricaturas del presidente estadounidense George W. Bush (Calogero y Mullen, 2008. Pág. 107).

El último de los artículos citados es "The Erotic and the Vulgar: Visual Culture and Organized Labor's Critique of U.S. Hegemony in Occupied Japan" de Cristopher Gerteis. Este estudio analiza la critica realizada por los sindicatos japoneses a través del humor gráfico de la hegemonía estadounidense tras la Segunda Guerra Mundial (Gerteis, 2007. Pág. 3). De este modo el artículo toma el humor gráfico como una fuente para realizar un estudio de tipo histórico.

Estas cifras implican que el campo de la Comunicación apenas recibe el 8\% de todas las citas obtenidas por los artículos que abordan el humor gráfico (ver Figura 6)

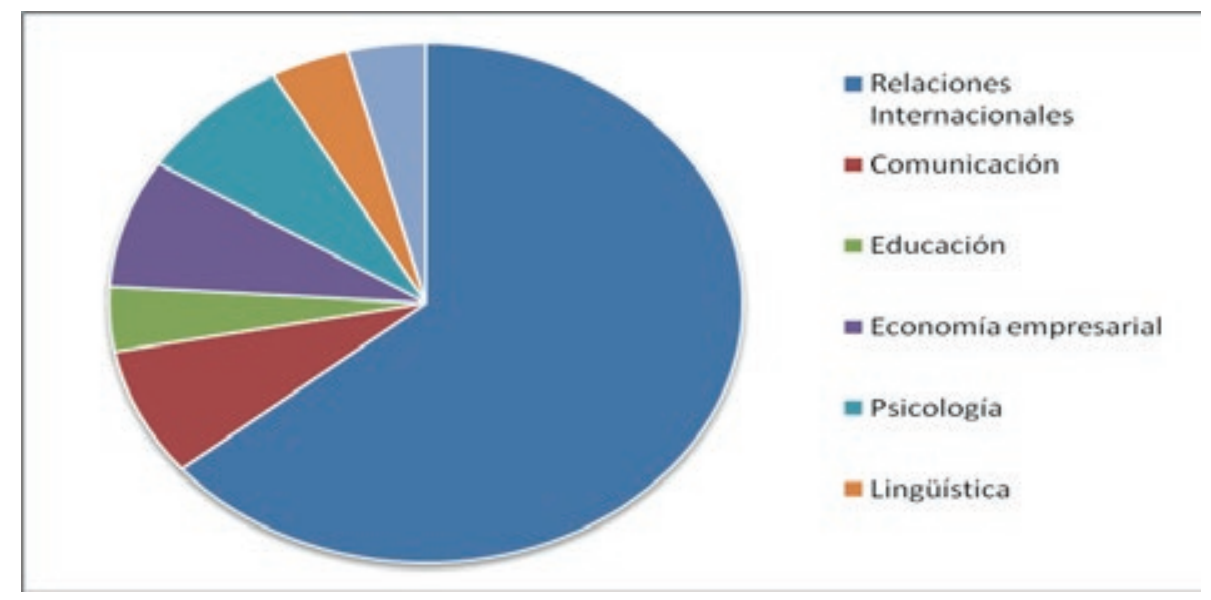

Figura 6. Porcentaje de citas recibidas por área de conocimiento de la revista en la que se publican los artículos analizados. Fuente: ISI Web of Knowledge 


\section{CONCLUSIONES}

Existen síntomas positivos del estado de la investigación en ciencias sociales: los catorce artículos recogidos en una base de datos de prestigio como el ISI Web of Knowledge constituyen un resultado suficientemente amplio para señalar su consolidación como objeto de estudio de interés. Sin embargo, aunque entre los autores aparece un total de diez académicos distintos tan sólo cuatro publican más de un artículo. Este dato indica que la producción científica en torno al humor gráfico es más bien fruto de investigaciones o esfuerzos individuales. Para acabar de fortalecerse, sería necesaria la existencia de proyectos o equipos de investigación que utilizasen el humor gráfico como fuente de sus estudios. La generalización de proyectos o de iniciativas con más continuidad contribuiría a superar ese panorama de trabajos e investigaciones aisladas y favorecer la consolidación de este objeto de estudio $^{4}$

Por otro lado, de toda la variedad de temas que son abordados por los estudios de la muestra tan sólo tres o cuatro asuntos despiertan la atención de otros estudios. En este sentido reciben un mayor número de citas aquellos artículos que tratan asuntos o personajes de interés global en la agenda mediática. Es el caso de los artículos de Calogero y Mullen sobre la imagen del presidente Bush y, sobre todo, del trabajo de Dodds acerca del tratamiento de la "Guerra contra el Terror". Otros trabajos similares acerca de asuntos más locales como el de Aliefendioglu y Yetin sobre Chipre, los artículos de Hammet sobre Zimbabwe o los estudios de Segado sobre la transición española a la democracia no han conseguido recibir ninguna cita. Tan sólo artículos vinculados al área anglosajona, como es el caso del trabajo de Gerteis sobre la ocupación estadounidense de Japón, han sido mencionados en otras investigaciones.

En este sentido los estudios de Comunicación sobre humor gráfico se benefician de abordar temas que no se ciñen necesariamente a la actualidad concreta de un país. Así lo demuestra el interés despertado por los estudios sobre recepción del humor gráfico de Elisabeth El Refaie. Aún así estos temas intemporales y globales no pueden superar en número de citas a aquellos estudios que se vinculan a asuntos privilegiados por la agenda mediática internacional.

Independientemente del área concreta de las ciencias sociales de la que surja, la producción científica sobre humor gráfico que obtiene un mayor impacto es la que se aleja de temas locales. Para obtener un mayor número de citas debe abordar asuntos atemporales (el proceso de recepción o de construcción del significado) o bien temas

\footnotetext{
4 Sin pretender hacer un estudio extenso de los proyectos de investigación sobre humor gráfico existentes en el E.E.E.S. merece la pena citar algunas iniciativas que se han dado ese sentido en nuestro sentido, como es el caso del proyecto "La risa valenciana o la saga del doctor Cudol. Les publicacions periòdiques d'humor a la Comunitat Valenciana (1810-2006)", con el código GV/2007/069, financiado por la Generalitat Valenciana, que ha arrojado resultados firmados por Enric Bordería Ortiz, Josep Lluís Gómez Mompart y Francesc Andreu Martínez Gallego
} 
incluidos en el repertorio mediático global, no circunscrito a un área geográfica limitada.

\section{REFERENCIAS}

Aliefendioglu, H. \& Yetin, D. (2011). Don't take it personally, it's just a joke: The masculine media discourse of jokes and cartoons on the Cyprus issue. Revista Women studies international forum, 2, (34), 101-111.

Bounegru, L. \& Forceville, C. (2011). Metaphors in editorial cartoons representing the global financial crisis. Visual Comunication, 2, (10), 209-229.

Bozal, V. (1989). El siglo de los caricaturistas. Madrid: Grupo 16.

Calogero, R. M. \& Mullen, B. (2008). About face: Facial prominence of George W. Bush in political cartoons as a function of war. Revista The Leadership Quarterly, 1, (19), 107116.

Castillo Esparcia, A. \& Ruiz Mora, I. (2011). Las revistas científicas de Comunicación en Latindex. En Fonseca, M. C.: Acceso y visibilidad de las revistas científicas españolas de Comunicación (pp. 8-22). Sociedad Tenerife: Latina de Comunicación Social.

Dodds, K. (2007). Steve Bell's eye: Cartoons, geopolitics and the visualization of the 'War on terror'. Revista Security Dialogue, 38, (2),157-177.

El Refaie, Elisabeth (2009). Multiliteracies: How readers interpret political cartoons. Revista Visual Comunication, 2 (8), 181-205.

El Refaie, Elisabeth \& Hörschelmann, K. (2010). Young people's readings of a political cartoon and the concept of multimodal literacy. Revista Discourse: Studies in the Cultural Politics of Education, 2, (31), 195-207.

Gerteis, C. (2007). The Erotic and the Vulgar: Visual Culture and Organized Labor's Critique of U.S. Hegemony in Occupied Japan. Revista Critical Asian Studies, 1, (39), 334.

Guitart Coria, M. B. \& Flores Martínez (2003). Humor gráfico para la enseñanza y el aprendizaje del azar. Revista Suma: Revista sobre Enseñanza y Aprendizaje de las matemáticas, (42), 81-89.

Hammet, D.(2011). Resistence, Power and Geopolitics in Zimbabwe. Revista Area, 2 (43),202-210.

Hammet, D. \& Mather, C. (2011).Beyond decoding: Political Cartoons in the Classroom. Revista Journal of geography in higher education, 3, (35),103-119. 
Lindholm, K. \& Olsson, E K. (2011). Crisis Communication as a Multilevel Game: The Muhammad Cartoons from a Crisis Diplomacy Perspective. The International Journal of Press/Politics, 16, (2), 254-271.

Llera, J. A. (2003). El humor verbal y visual de La Codorniz. Madrid: CSIC.

Marín Arrese, J. I. (2008). Cognition and culture in political cartoons. Intercultural pragmatics, 1, (5),1-18.

Martínez Nicolás, M. \& Saperas Lapiedra, E. (2011). La investigación sobre Comunicación en España (1998-2007). Análisis de los artículos publicados en revistas científicas. Revista Revista Latina de Comunicación Social, (66). Recuperado el 23 de enero del 2011, de

http://www.revistalatinacs.org/11/art/926_Vicalvaro/05_Nicolas.html.

Segado Boj, F. (2008). Elenco de una nueva etapa: los protagonistas de la transición en las viñetas de la prensa diaria. Revista Historia y comunicación social, (13), 167-178.

Segado Boj, F. (2008). Una pesadilla obsesiva: la crisis económica y la transición española a la democracia a través del humor gráfico (1974-1977). Revista Comunicación y Sociedad, 21, (2),141-169.

Segado Boj, F. (2009). El brazo informativo del gobierno: TVE en el humor gráfico de la prensa diaria de la transición (1974-1977). Revista Estudios sobre el mensaje periodístico, (15), 491-501.

Tubau, I. (1987).El humor gráfico en la prensa del franquismo. Barcelona: Mitre.

Zeman, Z. (1987). Heckling Hitler. Caricatures of the third reich.. Hanover: University Press of New England.

\section{Francisco Segado-Boj}

Licenciado en Periodismo por la Universidad Complutense de Madrid (2002) y Doctor por la misma universidad (2008) en el Departamento de Historia de la Comunicación Social. Su tesis doctoral aborda la representación de la transición española a la democracia en el humor gráfico en la prensa diaria. El humor gráfico constituye su principal línea de investigación. Sobre este tema ha publicado artículos en las principales revistas españoles del área de Comunicación y del área de Historia. En la actualidad también participa en distintos proyectos sobre la imagen de diferentes colectivos en los medios audiovisuales, concretamente en la televisión. 